\title{
Prevalence of depression, anxiety and quality of life among North Indian polycystic ovary syndrome women: evidence from a prospective observational study
}

\author{
Sana Habib ${ }^{1}$, Arifa Anwar ${ }^{2}$, Farazul Hoda ${ }^{1}$, Rishabh Verma ${ }^{1}$, M. Akhtar ${ }^{1}$, Abul Kalam Najmi ${ }^{1 *}$
}

\begin{abstract}
${ }^{1}$ Department of Pharmacology, School of Pharmaceutical Education and Research, Jamia Hamdard, New Delhi, India ${ }^{2}$ Department of Obstetrics and Gynecology, Hamdard Institute of Medical Sciences and Research (HIMSR), Jamia Hamdard, New Delhi, India
\end{abstract}

Received: 29 September 2021

Revised: 28 October 2021

Accepted: 29 October 2021

*Correspondence:

Dr. Abul Kalam Najmi,

Email: aknajmi@jamiahamdard.ac.in

Copyright: (C) the author(s), publisher and licensee Medip Academy. This is an open-access article distributed under the terms of the Creative Commons Attribution Non-Commercial License, which permits unrestricted non-commercial use, distribution, and reproduction in any medium, provided the original work is properly cited.

\begin{abstract}
Background: Polycystic ovary syndrome (PCOS) is a common heterogeneous gynaecological endocrine disorder characterized by clinical features including oligo-amenorrhea/ovulatory dysfunction, hyperandrogenism and polycystic ovarian morphology. PCOS increases the risk of depression and anxiety which leads to poor quality of life. Aim of the study were to determine the prevalence of anxiety and depression among women suffering from PCOS and to determine the quality of life (QOL) in PCOS women.

Methods: The study was prospective, observational, non-interventional and questionnaire-based. 192 women with PCOS voluntarily helped in filling the questionnaires consisting of questions using PHQ-9 for depression, GAD-7 for anxiety, SF-12 for general health and PCOSQ-50 for disease-specific domains. All data were recorded in pre-designed case record forms and analysis of data was done using different statistical methods.

Results: Majority of PCOS women were either overweight or obese. Based on PHQ-9 20\% of women was suffering with major depression and based on GAD-7, 25\% with major anxiety. It is found that psychosocial and emotional domain and coping domain of PCOSQ-50 is significant in patients with major depression and major anxiety. Significant effects were seen on their general health as per SF-12 domain. Lack of physical exercise was found in $83 \%$ of women. Conclusions: PCOS is a complex disease which decreases the overall quality of life. Therefore, treatment of PCOS women should include psychological counselling along-with with medication, especially in obese PCOS women. Women should be educated with the benefits of lifestyle modification in PCOS.
\end{abstract}

Keyword: PCOS, Anxiety, Depression, QOL

\section{INTRODUCTION}

Polycystic ovarian syndrome (PCOS) is a complex endocrine disorder of genetic origin, which is usually characterized by disorders in the menstrual cycle, infertility, metabolic syndrome and high level of male hormones. This change in hormones also leads to hirsutism, acne and alopecia. ${ }^{1-3}$ In the western world, PCOS is a common endocrine disorder and tends to affect $5-10 \%$ of women. ${ }^{4}$ In India, $3.7 \%-22.5 \%$ of women are affected by it as per Indian infertility society. ${ }^{5}$ Several health risks are associated with PCOS, among which the most common are obesity, insulin resistance, type 2 diabetes and changes in lipid profile. ${ }^{6-8}$ Possible health risks include cardiovascular disease (CVD) and associated risk factors. ${ }^{9,10}$

Women with PCOS have also been reported to have a higher psychological burden. They experience high rates of anxiety and depression as compared women in the general population. ${ }^{11}$ It is thus suggested that, by both 
international and Indian authorities, women with PCOS must undergo a psychological evaluation, especially for anxiety and depression. ${ }^{5}$ PCOS is not only associated with metabolic consequences but may also influence the quality of life and mental health due to psychological consequences of infertility and other symptoms may also have a negative impact on the quality of life. ${ }^{12,13}$ The incidence of depression in the general population is comparatively lesser (4-6\%) than PCOS women, which is much higher (14-67\%). ${ }^{7}$ Additionally, PCOS women are also characterized by a higher incidence of other psychological disorders like anxiety, eating disorders and substance abuse. ${ }^{9}$ Two such studies on Indian population investigated the prevalence of anxiety and depression among women with PCOS. The study reported that $28 \%$ and $39 \%$ anxiety was prevalent in the two studies respectively. And $11 \%$ and $25 \%$ of women were found to have depression. ${ }^{14,15}$

Despite these available data, there are no current recommendations for the need of psychological consultation in women with PCOS. There is a lack of data available from Indian settings, regarding the depression and anxiety affecting the quality of life in the patient having PCOS with or without co-morbidity/metabolic disorders.

\section{METHODS}

\section{Study design}

The study was prospective, observational and questionnaire-based. Single-center study was carried out in the OPD of gynecology and obstetrics department of HAH-C hospital, Jamia Hamdard, New Delhi, India from 15 December, 2017 to 14 April, 2018. Clinical evaluation and diagnosis of PCOS was done as per Rotterdam criteria 2003 , which is two out of a) oligo/anovulation and b) clinical and/or biochemical signs of hyperandrogenism and c) Polycystic ovaries. ${ }^{19}$

The study protocol was approved by Jamia Hamdard institutional ethics committee (JHIEC), New Delhi, India. This study was carried out in full compliance with the declaration of Helsinki. ${ }^{20}$ Written informed consent was obtained from all of the participants voluntarily. All participants were assured of their confidentiality and the anonymity of their identity.

\section{Study population}

Patient selection was carried out on the basis of predefined inclusion and exclusion criteria. Only participants those meeting all the following criteria were included in the study a) Female patients suffering from PCOS with or without co-morbidity between aged grouped 18-40 years and $b$ ) Patients who provided written and signed informed consent prior to initiation of the study. In our study we excluded who were not suffering from PCOS, pregnant women, and those women who were not willing to provide signed informed consent form.

We used modified Kuppuswamy's socioeconomic scale to assess the economic status of the patients. According to this scale, socioeconomic status was categorised into five subscales (upper, upper middle, lower middle, upper lower, and lower) on the basis of education, occupation of head of family, and monthly income of family.

\section{Questionnaire used}

PHQ-9: It is a standard questionnaire which assesses depression amongst the patient. It contains 9 questions which related the signs and symptoms of depression.

$G A D-7:$ It is a standard questionnaire which assesses anxiety amongst the patient. It contains 7 questions related to signs and symptoms of anxiety.

$S F-12$ : It is a standard version 2 questionnaire. It is used to assess general health of the patient having PCOS. It contains 12 questions related to both physical and mental health.

PCOSQ-50: It is a disease specific pre-validated questionnaire which assesses the disease specific problems affecting quality of life of patients. It contains 6 domains in it containing psychological and emotional, fertility, sexual function, obesity and menstrual disorders, hirsutism disorders and coping, among which Fertility and sexual dysfunction domains was administered to married patients only.

\section{Data collection}

Data were collected based on standardized interviews performed by the research team which was supported by qualified nurses. A written informed consent was obtained, and the participants were informed that they were free to refuse, participate or withdraw at any time without any disadvantage or prejudice. Parents or legal guardians of participants younger than 18 years provided written informed consent on behalf of the participants.

\section{Statistical analysis}

The data were pooled and statistically analysed using IBM SPSS 20 software. The sociodemographic profile and the prevalence of the different symptoms of PCOS, anxiety, and depression have been expressed in terms of frequency and percentages. Chi-square test was used to evaluate the statistical significance of differences among proportions of categorical data.

\section{RESULTS}

One hundred and nighty two women diagnosed with PCOS from the gynecology and obstetrics department of HAH-C hospital, Jamia Hamdard, New Delhi, India were included 
in the study for evaluation. These females belonged in the age group of 18-40 years. The sociodemographic profile of the population is presented in Table 1 . The $99(51.56 \%)$ of 192 women were found to be obese, waist hip ratio (WHR) ratio was found to be more than 0.85 in $75.65 \%$ of the population. 104 patients were evaluable for fertility and 27 of them were assessed to be infertile. The 190 patients were found with polycystic ovary, after undergoing ultrasound and have irregular menses. Other prevalent symptoms were hirsutism (in 82 patients) and acne (in 49 patients). More characteristic attributes of the disease in the population are presented in Table 2.

Amongst the enrolled population, a lack of physical exercise (159 of 192) was also observed. The 58 patients were found to have more of a junk diet and 74 patients experience disturbed and/or late-night sleep. More lifestyle-related attributes are presented in Table 3.

\section{Prevalence of anxiety and depression}

Of the 192 women enrolled in study 39 (20\%) were found to be in major depression according to the PHQ-9 scale (PHQ score $\geq 10$ for major depression). It was observed that $61.5 \%$ (24 women) of the women with major depression were obese while $49 \%(n=74)$ of the women without major depression was obese (Table 4). 53.8\% (21 women) were found to be at the risk of developing a behavioural eating disorder in women with major depression, while $42.4 \%$ (65 women) were at the same risk in the population without major depression. Major anxiety (according to PHQ-9) was also found to be more, $71.7 \%$ (28 women), in women with major depression than women without major depression $(7.1 \%)$.

About 25\% (47 women) of the population were found to have major anxiety, according to GAD 7 scale (GAD score $\geq 10$ corresponds to major anxiety). It was observed that $53.1 \%$ ( 25 women) of women with major anxiety was obese while $51 \%(\mathrm{n}=74)$ of women without major anxiety were obese (Table 5). 51\% (24 women) were found to be at the risk of developing a behavioural eating disorder in women with major anxiety, while $42.7 \%$ (62 women) were at the same risk in the population without major anxiety. Major depression (according to GAD-7) was also found to be more, $60 \%$ ( 28 women), in women with major depression than women without the major depression $(7.5 \%)$.

Quality of life of women with PCOS, analysing general, psychological and sexual health, shows in the Table 4 and 5.

Table 1: Socio-demographic characters of the study population, $(n=192)$.

\begin{tabular}{|lll|}
\hline Distribution of patients & No. of patients & Percentage (\%) \\
\hline Age distribution (years) & 32 & 16.67 \\
\hline $18-20$ & 95 & 49.48 \\
\hline $21-25$ & 49 & 25.52 \\
\hline $26-30$ & 12 & 6.25 \\
\hline $31-35$ & 4 & 2.08 \\
\hline $36-40$ & & \\
\hline Education & 7 & 3.65 \\
\hline Illiterate & 9 & 4.68 \\
\hline Middle school & 33 & 17.19 \\
\hline High school & 41 & 21.35 \\
\hline Higher secondary school & 69 & 35.94 \\
\hline Graduate degree & 33 & 17.19 \\
\hline Post graduate degree & & \\
\hline Socioeconomic status & 11 & 5.73 \\
\hline Lower & 55 & 28.65 \\
\hline Upper lower & 40 & 20.83 \\
\hline Lower middle & 71 & 36.98 \\
\hline Upper middle & 15 & 7.81 \\
\hline Upper & & 3.65 \\
\hline Category of BMI $\left(\mathbf{k g} / \mathbf{m}^{2}\right)$ & 7 & 44.79 \\
\hline Lean (BMI<18) & 86 & 24.48 \\
\hline Normal weight (BMI=18-22.99) & 47 & 25.52 \\
\hline Overweight (BMI=23-27.4) & 49 & 1.56 \\
\hline Obese (BMI=27.5-37.5) & 3 & 45.83 \\
\hline Extremely obese (BMI>37.5) & & 54.17 \\
\hline Marital status & 88 & \\
\hline Single & 104 & \\
\hline Married & & \\
\hline & & \\
\hline
\end{tabular}


Table 2: Anthropometric measurement of the patients.

\begin{tabular}{|ll|}
\hline Anthropometric measurements & Mean \pm SD \\
\hline Height $(\mathbf{m})$ & $1.54 \pm 0.05$ \\
\hline Weight $(\mathbf{k g})$ & $59.5 \pm 11.9$ \\
\hline BMI $\left(\mathbf{k g} / \mathbf{m}^{2}\right)$ & $24.88 \pm 4.55$ \\
\hline Waist circumference $(\mathbf{c m})$ & $81.68 \pm 11.70$ \\
\hline Hip circumference $\mathbf{( c m )}$ & $35.6 \pm 3.69$ \\
\hline WHR & $0.90 \pm 0.006$ \\
\hline
\end{tabular}

Table 3: Characters of patients attributing to the diseases, $(n=192)$.

\begin{tabular}{|c|c|c|c|c|}
\hline Distribution of characters & No. of patients & Percentage (\%) & Mean \pm SD & P value \\
\hline \multicolumn{5}{|l|}{ BMI $\left(\mathrm{kg} / \mathrm{m}^{2}\right)$} \\
\hline Less than 23 (non-obese) & 93 & 44.44 & \multirow{2}{*}{$24.88 \pm 4.53$} & \multirow{2}{*}{$<0.0001$} \\
\hline 23 and more (Obese) & 99 & 51.56 & & \\
\hline \multicolumn{5}{|l|}{ Waist circumference $(\mathbf{c m})$} \\
\hline Less than 88 & 144 & 75 & \multirow{2}{*}{$81.68 \pm 11.70$} & \multirow{2}{*}{$<0.0001$} \\
\hline 88 and more & 48 & 25 & & \\
\hline \multicolumn{5}{|l|}{ WHR } \\
\hline Less than 0.85 & 45 & 23.44 & \multirow{2}{*}{$0.90 \pm 0.66$} & \multirow{2}{*}{$<0.0001$} \\
\hline 0.85 and more & 147 & 76.56 & & \\
\hline \multicolumn{5}{|l|}{ BP (mmHg) } \\
\hline Systole & & & $115.55 \pm 9.611$ & \\
\hline Diastole & & & $77.34 \pm 5.70$ & \\
\hline \multicolumn{5}{|l|}{ Menarche } \\
\hline Early menarche & 63 & 32.81 & \multirow{3}{*}{$13.35 \pm 1.40$} & \\
\hline Normal menarche & 119 & 61.98 & & \\
\hline Late menarche & 10 & 5.21 & & \\
\hline \multicolumn{5}{|l|}{ Infertility status, $(\mathrm{n}=104)$} \\
\hline Yes & 77 & 74.04 & & \\
\hline No & 27 & 25.96 & & \\
\hline \multicolumn{5}{|l|}{ PCOD status } \\
\hline Polycystic ovary in USG & 190 & 98.95 & & \\
\hline $\begin{array}{l}\text { Oligomenorrhoea/ } \\
\text { Irregular menses }\end{array}$ & 190 & 98.95 & & \\
\hline Hirsutism & 82 & 42.17 & & \\
\hline Acne & 49 & 25.52 & & \\
\hline Presence of comorbid & 99 & 51.56 & & \\
\hline
\end{tabular}

Table 4: Characters attributing impact to lifestyle of patients, $(n=192)$.

\begin{tabular}{|lll|}
\hline Distribution of characters & No. of patients & Percentage (\%) \\
\hline Exercise & & 17.19 \\
\hline Yes & 33 & 82.81 \\
\hline No & 159 & 53.65 \\
\hline Diet & 103 & 30.21 \\
\hline Normal diet & 58 & 16.14 \\
\hline Junk diet & 31 & 61.46 \\
\hline Dieting behaviour & & 38.54 \\
\hline Sleep pattern & 118 & 13.02 \\
\hline Normal & 74 & 83.85 \\
\hline Disturbed/ Late night & & 3.13 \\
\hline Sleep duration (Hours) & 25 & 161 \\
\hline Less than 6 & 6 & \\
\hline 6-8 & & \\
\hline More than 8 & & \\
\hline
\end{tabular}


Table 5: Comparison of clinical features between women with major depression (PHQ-9 score $\geq 10$ ) and without major depression (PHQ-9 score $<10)$.

\begin{tabular}{|c|c|c|c|}
\hline Variables & With major depression, (n=39) & Without major depression, (n2=153) & P value \\
\hline \multicolumn{4}{|l|}{ BMI $\left(\mathrm{kg} / \mathrm{m}^{2}\right)$} \\
\hline Non-obese & 15 & 78 & \multirow{2}{*}{0.1} \\
\hline Obese & 24 & 75 & \\
\hline \multicolumn{4}{|l|}{ Marital status } \\
\hline Single & 15 & 73 & \multirow{2}{*}{0.3} \\
\hline Married & 24 & 80 & \\
\hline Infertility & 19 & 58 & 0.4 \\
\hline Hirsutism & 21 & 61 & 0.1 \\
\hline \multicolumn{4}{|l|}{ Have eating disorder } \\
\hline At risk of eating disorder & 13 & 10 & 0.05 \\
\hline $\begin{array}{l}\text { At risk of behavioral eating } \\
\text { disorder }\end{array}$ & 21 & 65 & 0.2 \\
\hline At risk of dementia & 16 & 51 & 0.3 \\
\hline Have comorbidities & 26 & 73 & 0.05 \\
\hline Major anxiety & 28 & 11 & 0.001 \\
\hline SF-12 & Mean \pm SD & Mean \pm SD & \\
\hline Physical functioning (PF) & $45.51 \pm 30.81$ & $76.14 \pm 29.01$ & 0.001 \\
\hline Role physical (RP) & $41.34 \pm 31.56$ & $69.52 \pm 26.04$ & 0.001 \\
\hline Body pain (BP) & $61.53 \pm 31.33$ & $79.90 \pm 24.17$ & 0.001 \\
\hline General health $(\mathrm{GH})$ & $32.05 \pm 25.22$ & $53.82 \pm 21.57$ & 0.001 \\
\hline Vitality (VT) & $34.61 \pm 22.66$ & $55.22 \pm 22.14$ & 0.001 \\
\hline Social functioning (SF) & $58.33 \pm 27.10$ & $88.23 \pm 18.81$ & 0.001 \\
\hline Role emotional (RE) & $31.73 \pm 26.41$ & $61.84 \pm 26.96$ & 0.001 \\
\hline Mental health (MH) & $41.67 \pm 19.09$ & $65.19 \pm 21.60$ & 0.001 \\
\hline Physical health (PCS) & $43.34 \pm 10.95$ & $50.30 \pm 7.74$ & 0.001 \\
\hline Mental health (MCS) & $33.47 \pm 9.13$ & $45.05 \pm 10.06$ & 0.001 \\
\hline \multicolumn{4}{|l|}{ PCOSQ-50 } \\
\hline Psychosocial and emotional & $2.85 \pm 0.67$ & $3.57 \pm 0.53$ & 0.001 \\
\hline Fertility, $(n=104)$ & $1.74 \pm 1.57$ & $1.74 \pm 1.81$ & 0.9 \\
\hline Sexual function, $(n=104)$ & $2.01 \pm 1.79$ & $2.21 \pm 2.20$ & 0.5 \\
\hline $\begin{array}{l}\text { Obesity and menstrual } \\
\text { disorders }\end{array}$ & $2.93 \pm 0.91$ & $3.38 \pm 0.82$ & 0.001 \\
\hline Hirsutism disorders & $3.33 \pm 1.44$ & $3.80 \pm 1.29$ & 0.05 \\
\hline Coping & $3.19 \pm 0.75$ & $3.64 \pm 0.79$ & 0.001 \\
\hline
\end{tabular}

Table 6: Comparison of clinical features between women with major anxiety (GAD score $\geq 10$ ) and without major anxiety (GAD score $<10)$.

\begin{tabular}{|c|c|c|c|}
\hline Variables & With major anxiety, $\left(n_{1=47)}\right.$ & Without major anxiety, $\left(n_{2}=145\right)$ & P value \\
\hline \multicolumn{4}{|l|}{ BMI $\left(\mathrm{kg} / \mathrm{m}^{2}\right)$} \\
\hline Non-obese & 22 & 71 & \multirow{2}{*}{0.7} \\
\hline Obese & 25 & 74 & \\
\hline \multicolumn{4}{|l|}{ Marital status } \\
\hline Single & 18 & 70 & \multirow{2}{*}{0.2} \\
\hline Married & 29 & 75 & \\
\hline Infertility & 4 & 23 & 0.07 \\
\hline Hirsutism & 22 & 60 & 0.5 \\
\hline \multicolumn{4}{|l|}{ Have eating disorder } \\
\hline At risk of eating disorder & 7 & 16 & 0.4 \\
\hline $\begin{array}{l}\text { At risk of behavioral eating } \\
\text { disorder }\end{array}$ & 24 & 62 & 0.3 \\
\hline At risk of dementia & 17 & 50 & 0.8 \\
\hline Have comorbidities & 26 & 73 & 0.5 \\
\hline Major depression & 28 & 11 & 0.001 \\
\hline
\end{tabular}




\begin{tabular}{|llll|}
\hline Variables & With major anxiety, $\left(\mathbf{n}_{1}=47\right)$ & Without major anxiety, $\left(\mathbf{n}_{2}=\mathbf{1 4 5}\right)$ & P value \\
\hline SF-12 & Mean \pm SD & Mean \pm SD & \\
\hline Physical functioning $(\mathrm{PF})$ & $55.85 \pm 32.64$ & $74.48 \pm 30.25$ & 0.001 \\
\hline Role physical (RP) & $51.06 \pm 32.95$ & $67.93 \pm 27.07$ & 0.001 \\
\hline Body pain (BP) & $65.95 \pm 30.58$ & $79.48 \pm 24.59$ & 0.002 \\
\hline General health (GH) & $35 \pm 24.78$ & $54.06 \pm 1.81$ & 0.001 \\
\hline Vitality (VT) & $42.02 \pm 25.05$ & $53.96 \pm 22.56$ & 0.002 \\
\hline Social functioning (SF) & $61.17 \pm 26.99$ & $88.96 \pm 18.37$ & 0.001 \\
\hline Role emotional (RE) & $32.44 \pm 23.26$ & $63.27 \pm 27.21$ & 0.001 \\
\hline Mental health (MH) & $42.02 \pm 18.13$ & $66.37 \pm 21.37$ & 0.001 \\
\hline Physical health (PCS) & $46.67 \pm 11.56$ & $49.61 \pm 7.78$ & 0.05 \\
\hline Mental health (MCS) & $33.27 \pm 8.27$ & $45.75 \pm 9.87$ & 0.001 \\
\hline PCOSQ-50 & $2.88 \pm 0.61$ & $3.60 \pm 0.53$ & 0.001 \\
\hline Psychosocial and emotional & $1.53 \pm 1.37$ & $1.81 \pm 1.87$ & 0.3 \\
\hline Fertility, $(\mathrm{n}=104)$ & $1.99 \pm 1.76$ & $2.22 \pm 2.23$ & 0.5 \\
\hline Sexual function, $(\mathrm{n}=104)$ & $3.02 \pm 0.86$ & $3.37 \pm 0.83$ & 0.01 \\
\hline $\begin{array}{l}\text { Obesity and menstrual } \\
\text { disorders }\end{array}$ & $3.57 \pm 1.41$ & $3.74 \pm 1.31$ & 0.4 \\
\hline Hirsutism disorders & $3.10 \pm 0.65$ & $3.70 \pm 0.79$ & 0.001 \\
\hline Coping & & \\
\hline * $<0.05=$ significant, **p<0.01=more significant, ***p<0.001=highly significant & & \\
\hline
\end{tabular}

\section{DISCUSSION}

The results of our study suggest that the majority of women with PCOS are obese $(51.56 \%)$ and have abdominal obesity $(76.56 \%)$. Menstrual irregularities (98.95\%), acne (25.52\%) and hirsutism (42.17\%) were the important issues reported by women with PCOS. Similar finding was also seen in the study done by Cronin et al reported that the main problems for the women affected by PCOS were hirsutism $(90 \%)$, obesity $(84 \%)$, menstrual disorders $(82 \%)$, and difficulty maintaining their body weight $(80 \%) .{ }^{21}$

In this study, women with PCOS are at high risk of mental disorders and reduced QOL as more than half of the study population experienced depression and anxiety scored from mild to severe. Approximately $20 \%$ and $25 \%$ women are having major depression and major anxiety respectively which forms the cut-off for consulting psychological and psychiatric professionals. This study found strong association between depression and anxiety as the result is highly significant $(\mathrm{p}<0.001)$. This finding is similar to another recent study carried out in by StapinskaSyniec et al and Sulaiman et al. ${ }^{3,12}$ Our findings correspond with the other literature also, where the prevalence of depressive symptoms in women with PCOS ranges from $14 \%$ to $67 \% .{ }^{18}$ The most common mental problem that affected half of the study group was depression. Several other studies have also confirmed the increased incidence of depression in women with PCOS compared to ageadjusted controls. ${ }^{22,23}$ Women with PCOS with major depression in our study population had excess body weight that is either they are overweight or obese. Similar is the result for anxiety in this study that only $25(13.02 \%)$ obese women correspond with major anxiety.
Prevalence of infertility status was found higher amongst women who lead to psychological problems at some stage of their life. ${ }^{24}$ Infertility causes family tensions and problems at work, while obesity in PCOS women is responsible for a general state of depression and dissatisfaction..$^{25,26}$ Infertility is a frequently encountered problem in patients with PCOS that could contribute to an increase in the incidence of depression. Deeks et al showed that $64 \%$ of fertile and $81 \%$ of infertile women with PCOS reported moodiness. The ratios of clinically confirmed depression diagnosis were significantly lower (28\% vs. $40 \%$, respectively). ${ }^{27}$ In a population of 1741 women with PCOS, primary infertility occurred in one in two, whereas secondary infertility in every fourth participant. ${ }^{28}$ Similar findings were present in this study also but majority of them were having primary infertility. Studies indicate that women with PCOS who have been unable to get pregnant or have experienced recurrent miscarriages often complained of reduced libido, which constituted additional source of sexual stress. ${ }^{12}$ However, the effect of infertility on mood impairment remains undetermined. Dokras et al. did not find any significant discrepancies in the BDI scores between women with and without infertility. ${ }^{6}$ Similarly, in a study carried out by Stapinska-Syniec et al infertility was identified in one-third of depressed women and the same proportion was observed in respondents with normal BDI scores, which suggest that infertility is not an isolated risk factor for depression in women with PCOS. ${ }^{29}$

The reduced quality of sexual life in women with PCOS is gaining recognition among researchers. ${ }^{30,31}$ In this study average score of sexual function do not indicate poor or negative result. In a study by Stapinska-Syniec et al approximately a third of all respondents assessed the level of their sexual satisfaction as low and no relationship between the presence of hirsutism and low sexual 
satisfaction was found. ${ }^{29}$ In this study, no significant result is seen for sexual function domain of PCOSQ-50.

In this study population, majority of women have anxiety scored as mild, moderate or severe and $24.48 \%$ of the respondents reported with major anxiety disorders. Dokras et al reported similar results and stressed a significantly higher incidence of anxiety disorders in women with PCOS compared to the general population (14\% vs. $1 \%){ }^{6}$ In case of depression, majority of women were found to have mild to severe anxiety. Obesity and menstrual disorder domain of PCOSQ-50 was found highly related to major depressive women. Similarly, in this study also, women reported eating disorder association with depression but not anxiety. Risk of eating disorder and risk of behavioral eating disorder was more in patients having major depression. Therefore, it is suggested that these patients should consult professional help for further confirmation of eating disorder.

Obese women with PCOS, as well as women lacking adequate family support and dissatisfied with their sexual life may be the group that would benefit the most from such an intervention. ${ }^{32}$ Moreover, repeated evaluations of the patient's QOL and coping mechanisms over a long period of time could facilitate early diagnosis of psychological disturbances. ${ }^{33}$ Therefore, we would like to emphasize that the therapy of women with PCOS should be comprehensive and ideally include psychological and in some cases psychiatric consultations.

\section{Study strength}

Strength of the current study was absence of protocol violations and objectively measured primary outcome. Although this study contributes valuable evidence to the literature. Additionally, data gathered from this study will be useful for health policy maker and in formulation of national guidelines.

\section{Limitations}

Firstly, this is a prospective observation study without a single follow up. Secondly, actual correlation with anxiety and depression were not been identified.

\section{CONCLUSION}

In conclusion, our study found high prevalence of PCOS among patients visiting outpatient department. PCOS are increasing day by day which may have serious negative impact on women's health and affecting overall QoL. In order to combat this situation, promotion on large-scale involving women health educational interventions should be undertaken. High prevalence of depression, anxiety, dementia and eating disorder were found amongst the patients. Thus, an appropriate multidisciplinary approach towards psychological management, safety and efficacy of the treatment through health education and counselling should be implemented.
Funding: No funding sources

Conflict of interest: None declared

Ethical approval: The study was approved by the Institutional Ethics Committee

\section{REFERENCES}

1. Azziz R, Woods KS, Reyna R, Key TJ. The prevalence and features of the polycystic ovary syndrome in an unselected population. J Clin Endocrinol Metab. 2004;89(6):2745-9.

2. Ehrmann DA. Polycystic ovary syndrome. N Eng J Med. 2005;352(12):1223-36.

3. Sulaiman MA, Al-Farsi YM, Al-Khaduri MM. Psychological burden among women with polycystic ovarian syndrome in Oman: a case-control study. In j women's health. 2017;9:897.

4. Malik-Aslam A, Reaney MD, Speight J. The Suitability of Polycystic Ovary Syndrome-Specific Questionnaires for Measuring the Impact of PCOS on Quality of Life in Clinical Trials. Value in health, 2010;13(4):440-6.

5. Sonia M, Jain K, Talwar P, Prasad S, Dhorepatil B. Management of polycystic ovary syndrome in India. Fertility Sci Res. 2014;1:23.

6. Anuja D, Bochner M, Hollinrake E, Markham S, Van Voorhis B. Screening women with polycystic ovary syndrome for metabolic syndrome. Obstetri Gynecol. 2005;106(1):131-7.

7. Richard LS, Kunselman AR, Dodson WC, Dunaif A. Prevalence and predictors of risk for type 2 diabetes mellitus and impaired glucose tolerance in polycystic ovary syndrome: a prospective, controlled study in 254 affected women. J clin endocrinol metab. 1999;84(1):165-9.

8. Jan H, Bergh T, Berne C, Lithell H. Serum lipoprotein lipid profile in women with the polycystic ovary syndrome: relation to anthropometric, endocrine and metabolic variables. Clin endocrinol. 1994;41(1):46371.

9. Michael S, Kong C, Patel A, Unwin R, Jacobs HS. Ambulatory blood pressure profiles and plasminogen activator inhibitor (PAI-1) activity in lean women with and without the polycystic ovary syndrome. Clin endocrinol. 1996;45(5):623-9.

10. Kalpana L, Seifalian AM, Hardiman P. Impaired carotid viscoelastic properties in women with polycystic ovaries. Circulation. 2002;106(1):81-5.

11. Mattias M, Holte J, Landin-Wilhelmsen K, Dahlgren E, Johansson A. Women with polycystic ovary syndrome are often depressed or anxious-a case control study. Psychoneuroendocrinology. 2008;33(8):1132-8

12. Helena V, Svanberg AS, Ekselius L, Lundkvist O, Poromaa IS. Prevalence of psychiatric disorders in infertile women and men undergoing in vitro fertilization treatment. Human Reproduction. 2008;23(9):2056-63.

13. Barnard L, Ferriday D, Guenther N, Strauss B, Balen $\mathrm{AH}$, Dye L. Quality of life and psychological well 
being in polycystic ovary syndrome. Human reproduction. 2007;22(8):2279-86.

14. Upadhyaya, Kumar S, Sharma A, Agrawal A. Prevalence of anxiety and depression in polycystic ovarian syndrome. Int $\mathrm{J}$ Med Sci Public Health. 2016;5(4):681-3.

15. Arshad H, Chandel RK, M. Ashraf G, Dar MA, Rather YH. Prevalence of psychiatric disorders in patients with a diagnosis of polycystic ovary syndrome in Kashmir. Indian j psychol med. 2015;37(1):66-70.

16. Fauser, Bart CJM, Tarlatzis BC, Rebar RW, Legro $\mathrm{RS}$, Balen $\mathrm{AH}$ et al. Consensus on women's health aspects of polycystic ovary syndrome (PCOS): the Amsterdam ESHRE/ASRM-Sponsored 3rd PCOS Consensus Workshop Group. Fertility sterility. 2012;97(1):28-38.

17. Ndefo, Anadu U, Eaton A, Green MR. Polycystic ovary syndrome: a review of treatment options with a focus on pharmacological approaches. Pharmacy therapeutics. 2013;38(6):336.

18. Escobar-Morreale, Hector F. Polycystic ovary syndrome: definition, aetiology, diagnosis and treatment. Nature Reviews Endocrinol. 2018;14(5):270-84.

19. ESHRE, The Rotterdam, and ASRM-Sponsored PCOS Consensus Workshop Group. Revised 2003 consensus on diagnostic criteria and long-term health risks related to polycystic ovary syndrome. Fertility sterility. 2004;81(1):19-25.

20. Association WM, World Medical Association Declaration of Helsinki. Ethical principles for medical research involving human subjects. Bull World Health Organization. 2001;79(4):373.

21. Hahn, Susanne, Janssen OE, Tan S, Pleger K, Mann $\mathrm{K}$ et al. Clinical and psychological correlates of quality-of-life in polycystic ovary syndrome. Eur J Endocrinol. 2005;153(6):853-60.

22. Kumarapeli V, De Seneviratne RA, Wijeyaratne CN, Yapa RM, Dodampahala SH. A simple screening approach for assessing community prevalence and phenotype of polycystic ovary syndrome in a semiurban population in Sri Lanka. Am j epidemiol. 2008;168(3):321-8.

23. Blay, Luis S, Aguiar JVA, Passos IC. Polycystic ovary syndrome and mental disorders: a systematic review and exploratory meta-analysis. Neuropsychiatric disease treatment. 2016;12:2895.

24. Bhattacharya, Mohan S, Jha A. Prevalence and risk of depressive disorders in women with polycystic ovary syndrome (PCOS). Fertility sterility. 2010;94(1):3579.
25. Balen, Adam H, Morley LC, Misso M, Franks S. The management of anovulatory infertility in women with polycystic ovary syndrome: an analysis of the evidence to support the development of global WHO guidance. Human reproduction update. 2016;22(6):687-708.

26. Veltman-Verhulst, Susanne M, Boivin J, Eijkemans MJC, Fauser BJC. Emotional distress is a common risk in women with polycystic ovary syndrome: a systematic review and meta-analysis of 28 studies. Human reproduction update. 2012;18(6):638-51.

27. Deeks AA, Gibson-Helm ME, Paul E, Teede HJ. Is having polycystic ovary syndrome a predictor of poor psychological function including anxiety and depression? Human Reproduction. 2011;26(6):1399407.

28. Balen, Adam H, Conway GS, Kaltsas G, Techatraisak K, Manning PJ et al. Andrology: Polycystic ovary syndrome: the spectrum of the disorder in 1741 patients. Human reproduction. 1995;10(8):2107-11.

29. Stapinska-Syniec A, Grabowska K, SzpotanskaSikorska M, Pietrzak B. Depression, sexual satisfaction, and other psychological issues in women with polycystic ovary syndrome. Gynecol Endocrinol. 2018;34(7):597-600.

30. Maria E, Wijma K, Benzein E. "It is always on my mind": Women's experiences of their bodies when living with hirsutism. Health care for women int. 2009;30(5):358-72.

31. Ioannis ME, Messini CI, Anifandis G, Dafopoulos K. Polycystic ovaries and obesity. Best Practice Res Clin Obstetr Gynaecol. 2015;29(4):479-88.

32. Siew L, Smith CA, Costello MF, MacMillan F, Moran L. Barriers and facilitators to weight management in overweight and obese women living in Australia with PCOS: a qualitative study. BMC endocrine disorders. 2019;19(1):1-9.

33. Michael J, Fatehi F, Phillips-Chan ME, Richardson JR. Exploratory study of a screening measure for polycystic ovarian syndrome, quality of life assessment, and neuropsychological evaluation. BMC Women's Health. 2020;20(1):1-12.

Cite this article as: Habib S, Anwar A, Hoda F, Verma R, Akhtar M, Najmi AK. Prevalence of depression, anxiety and quality of life among North Indian polycystic ovary syndrome women: evidence from a prospective observational study. Int J Basic Clin Pharmacol 2021;10:1360-7. 